\title{
Functional Polymers: Synthesis and Polymerization of Vinyl and Methacrylate Monomers Containing the Pendant 1,3,5-Triphenyl-2-pyrazoline Group
}

\author{
Fujio IInUma, Hiroshi MikawA, and Yasuhiko SHIRoTA \\ Department of Applied Chemistry, Faculty of Engineering, Osaka University, \\ Yamadakami, Suita, Osaka 565, Japan.
}

(Received November 7, 1980)

\begin{abstract}
For the purpose of developing new functional polymers for potential use in imaging systems, the new vinyl and methacrylate monomers, 1,3-diphenyl-5-( $p$-vinylphenyl)-2pyrazoline and 1,3-diphenyl-5-( $p$-methacryloyloxyphenyl)-2-pyrazoline, were synthesized and polymerized by a free-radical method. The homopolymerization of these two monomers afforded vinyl and methacrylate polymers with $M_{n}=9,000-18,000$ and $M_{n}=10,000-25,000$, respectively. They were copolymerized with styrene and methyl methacrylate to give the corresponding copolymers. The free-radical polymerizability of the two monomers was found to be similar to that of $p$-methylstyrene and phenyl mathacrylate, respectively. The monomer synthesis, polymerization features, and characterization of the resulting polymers are described.

KEY WORDS Functional Polymer / Radical Polymerization / Radical Copolymerization / 1,3-Diphenyl-5-( $p$-vinylphenyl)-2-pyrazoline / 1,3Diphenyl-5-( $p$-methacryloyloxyphenyl)-2-pyrazoline / Styrene / Methyl Methacrylate / Poly[1,3-diphenyl-5-( $p$-vinylphenyl)-2-pyrazoline] / Poly[1,3diphenyl-5-( $p$-methacryloyloxyphenyl)-2-pyrazoline] /
\end{abstract}

Recently a variety of functional polymers have been prepared and their properties, functions, and applications have been extensively studied. Functional polymers are of value since they possess not only specific properties or functions but also processability due to their mechanical properties, thus offering an advantage in the fabrication of devices. On the other hand, blend systems where low-molecular-weight functional materials are dispersed in polymeric binders are also used for practical applications. For designing and developing functional polymers, it is of importance to understand polymer effects on the properties and functions, e.g., the enhancement, reduction or modulation of properties, or the appearance of novel properties in polymers.

The purpose of our investigation is to synthesize new functional polymers for potential use in electrophotography and other imaging systems and also to illuminate polymer effects on the physical properties and functions compared with low molecularweight model compounds. In the present study we have undertaken the synthesis of vinyl and methacrylate polymers containing the 1,3,5-triphenyl-2pyrazoline group as a pendant unit to the polymer backbone. 1,3,5-Triphenyl-2-pyrazoline (TPP) and its derivatives have been known to possess photoconducting property and some of them have recently found successful application as materials for use in electrophotography by being dispersed in a polymeric binder. ${ }^{1}$ These compounds are also known to be strongly fluorescent ${ }^{2,3}$ and have been patented as fluorescence whitening agents for wool and certain synthetic fibers. ${ }^{4}$ It is expected that polymers containing pendant TPP groups may be excellent photoreceptor materials in electrophotography. They may also be used as materials for colored image formation in the combination with polyhalogenated compounds on exposure. In addition, they will serve as suitable materials for studying polymer effects on the spectroscopic and photophysical properties as well as the photoconductive property, since the strong fluorescence characteristics of TPP and its derivatives can be used as 
probes for the spectroscopic studies and since extensive studies have been made of the spectroscopic and photophysical properties of TPP and its derivatives. ${ }^{2,3.5-7}$ The ready supercooling and glassforming properties of TPP and its derivatives ${ }^{6}$ will enable one to make comparative studies of the photoconductivity between TPP and polymers containing pendant TPP groups in their amorphous solids. The polymers containing pendant TPP groups have been prepared by the method of polymer reactions. That is, the polymer with a poly[1,5diphenyl-3-( $p$-vinylphenyl)-2-pyrazoline] structure has been prepared starting from polystyrene and its photoconductivity has been examined. ${ }^{8,9}$ Very recently, polymers with TPP in the polymer backbone have been prepared using $p$-chloromethylated polystyrene and shown to function as electrochromic materials. ${ }^{10}$ Compared with the polymer reaction method, the monomer synthesis and polymerization approach provides a route to vinyl polymers with regular incorporation of functional groups. This paper deals with the synthesis and polymerization of the new vinyl and methacrylate monomers, 1,3diphenyl-5-( $p$-vinylphenyl)-2-pyrazoline (VTPP) and 1,3-diphenyl-5-( $p$-methacryloyloxyphenyl)-2pyrazoline (MTPP). The present study has led to the first successful synthesis of vinyl and methacrylate polymers containing the TPP group as a pendant unit to the polymer backbone. The polymer effect on the spectroscopic and photophysical properties, and singlet excitation energy migration, in these polymers will be reported elsewhere. ${ }^{11}$

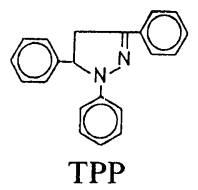

\section{EXPERIMENTAL}

\section{Materials}

Styrene, methyl methacrylate (MMA), methacryloyl chloride, fumaronitrile and diethyl fumarate were obtained commercially and purified by the ordinary manners. They were redistilled or resublimed in vacuo immediately before use. $p$-Divinylbenzene was prepared from commercially available divinylbenzene according to the literature ${ }^{12}: p$ bis(1,2-dibromoethyl)benzene, formed by the bromination of commercial divinylbenzene, was isolated, purified by recrystallizations from benzene and then debrominated with zinc dust in dioxane/water to give $p$-divinylbenzene, which was purified by distillation in vacuo, bp $41^{\circ} \mathrm{C}(0.6$ $\mathrm{mmHg})$, mp $28-29^{\circ} \mathrm{C}$ [lit. ${ }^{12}$ bp $40-43^{\circ} \mathrm{C}(0.7$ $\mathrm{mmHg}$ ), mp $25-26^{\circ} \mathrm{C}$. $\quad N$-Anilinobenzimidoyl chloride was prepared by the reaction of phosphorus pentachloride with $\beta$-benzoylphenylhydrazine which was prepared from benzoic anhydride on the treatment of phenylhydrazine, $\mathrm{mp} 129$ $130^{\circ} \mathrm{C}$ (lit. ${ }^{13} \mathrm{mp} 130^{\circ} \mathrm{C}$ ), 1,3-Diphenyl-5-( $p$-hydroxyphenyl)-2-pyrazoline was prepared by the acid-catalyzed reaction in ethanol between phenylhydrazine and $p$-hydroxybenzalacetophenone which had been prepared by the alkali-catalyzed reaction of $p$-hydroxybenzaldehyde with acetophenone in ethanol, mp $143-144.5^{\circ} \mathrm{C}$ (lit. ${ }^{14} \mathrm{mp} 146^{\circ} \mathrm{C}$ ), and recrystallized from ethanol-hexane $(1: 6)$. 2,2'Azobisisobutyronitrile (AIBN) was purified by recrystallizations from methanol, dried and stored in a refrigerator. Benzene, tetrahydrofuran and other solvents were purified and dried by the ordinary methods.

\section{1,3-Diphenyl-5-( p-vinylphenyl)-2-pyrazoline (VTPP)}

$p$-Divinylbenzene $(6.5 \mathrm{~g})$ and $N$-anilinobenzimidoyl chloride $(15 \mathrm{~g})$ were dissolved in $300 \mathrm{ml}$ benzene, and triethylamine $(6.6 \mathrm{~g})$ was added dropwise over a period of one hour to the solution at room temperature. After the reaction solution was allowed to stand for a day, the resulting triethylamine hydrochloride was removed by filtration. The filtrate was evaporated to dryness under reduced pressure. The crude product obtained was chromatographed over neutral alumina with benzene as an eluent, recrystallized three times from methanol and dried in vacuo to give VTPP $(3.5 \mathrm{~g})$, $\mathrm{mp} 132-133^{\circ} \mathrm{C}$ (pale yellow needle).

Anal. Calcd for $\mathrm{C}_{23} \mathrm{H}_{20} \mathrm{~N}_{2}$ : C, $85.14 \% ; \mathrm{H}, 6.23 \%$; $\mathrm{N}, 8.64 \%$. Found: C, $85.20 \%$; H, $6.25 \%$;, $8.56 \%$. $\mathrm{UV}, \lambda_{\max }$ in benzene, $362 \mathrm{~nm}(\log \varepsilon 4.30) ;{ }^{1} \mathrm{H}$ NMR in benzene- $d_{6} \delta, 2.62\left(1 \mathrm{H}, \mathrm{q}, \mathrm{CH}_{2}\right.$ in TPP ring, $\left.J_{\text {gem }}=17.5 \mathrm{~Hz}, J_{\text {vic }}=7.4 \mathrm{~Hz}\right), 3.14\left(1 \mathrm{H}, \mathrm{q}, \mathrm{CH}_{2}\right.$ in TPP ring, $\left.J_{\text {vic }}=12.4 \mathrm{~Hz}\right), 4.80(1 \mathrm{H}, \mathrm{q}, \mathrm{CH}$ in TPP ring), $5.04 .\left(1 \mathrm{H}, \quad \mathrm{q}\right.$, vinyl $\mathrm{CH}_{2}, \quad J_{\text {gem }}=1.1 \mathrm{~Hz}$, $\left.J_{\text {vic }}=11.0 \mathrm{~Hz}\right), \quad 5.52\left(1 \mathrm{H}, \quad \mathrm{q}, \quad\right.$ vinyl $\mid \mathrm{CH}_{2}$, $\left.J_{\text {vic }}=17.5 \mathrm{~Hz}\right), 6.48(1 \mathrm{H}, \mathrm{q}$, vinyl $\mathrm{CH})$, and 6.64 $7.76 \mathrm{ppm}(14 \mathrm{H}, \mathrm{m}$, phenyl ring protons); mass spectrum $m / e 324\left(\mathbf{M}^{+}\right)$. 


\section{1,3-Diphenyl-5-( p-methacryloyloxyphenyl)-2- pyrazoline (MTPP)}

A solution of $25 \mathrm{~g}$ of methacryloyl chloride and $37 \mathrm{~g}$ of 1,3-diphenyl-5-( $p$-hydroxyphenyl)-2-pyrazoline in $300 \mathrm{ml}$ of anhydrous tetrahydrofuran was stirred at room temperature and $30 \mathrm{ml}$ of triethylamine was added dropwise over three hours to the solution. The reaction solution was permitted to stand for a day, and then the resulting triethylamine hydrochloride was removed by filtration. The filtrate was evaporated to dryness under reduced pressure. The crude product obtained was purified by silicagel column chromatography with benzene as an eluent, followed by three recrystallizations from hexane, and dried in vacuo to give $25 \mathrm{~g}$ of MTPP, $\mathrm{mp} 115-116^{\circ} \mathrm{C}$ (pale yellow needle).

Anal. Calcd for $\mathrm{C}_{25} \mathrm{H}_{22} \mathrm{~N}_{2} \mathrm{O}_{2}: \mathrm{C}, 78.51 \% ; \mathrm{H}$, $5.80 \%$; N $7.32 \%$. Found: C, 78.20\%; H, 5.68\%; N, $7.29 \%$. UV, $\lambda_{\max }$ in benzene, $361 \mathrm{~nm}(\log \varepsilon 4.30) .{ }^{1} \mathrm{H}$ NMR in benzene- $d_{6}, \delta 1.82\left(3 \mathrm{H}, \mathrm{s}, \mathrm{CH}_{3}\right), 2.53(1 \mathrm{H}$, q, $\mathrm{CH}_{2}$ in TPP ring, $J_{\mathrm{gem}}=18.0 \mathrm{~Hz}, J_{\mathrm{vic}}=7.2 \mathrm{~Hz}$ ), $3.10\left(1 \mathrm{H}, \mathrm{q}, \mathrm{CH}_{2}\right.$ in TPP ring, $\left.J_{\text {vic }}=12.4 \mathrm{~Hz}\right), 4.73$ $\left(1 \mathrm{H}, \mathrm{q}, \mathrm{CH}\right.$ in TPP ring), $5.25\left(1 \mathrm{H}, \mathrm{d}\right.$, vinyl $\mathrm{CH}_{2}$, $\left.J_{\text {gem }}=1.1 \mathrm{~Hz}\right), 6.19\left(1 \mathrm{H}, \mathrm{d}\right.$, vinyl $\left.\mathrm{CH}_{2}\right)$, and $6.60-$ $7.76 \mathrm{ppm}(14 \mathrm{H}, \mathrm{m}$, phenyl ring protons); mass spectrum $m / e 382\left(\mathrm{M}^{+}\right)$.

\section{Polymerization Procedure}

Polymerizations were carried out in benzene and tetrahydrofuran (THF) for the VTPP and MTPP monomers, respectively. A polymerization solution containing the monomer and initiator in a glass tube $\left(1.5 \mathrm{~cm}\right.$ in diameter) was evacuated at $2 \times 10^{-2}$ Torr by means of several freeze-pump-thaw cycles, sealed off and then allowed to stand for an appropriate time in a thermostat maintained within $\pm 0.1^{\circ} \mathrm{C}$. The polymerization was stopped by chilling the polymerization solution and the solution was poured into a large volume of methanol to precipitate the polymers. The polymers were collected by filtration, washed repeatedly with methanol and dried in vacuo. Poly[1,3-diephenyl-5-( $p$ vinylphenyl)-2-pyrazoline] (PVTPP) and poly[1,3dipehnyl-5-( $p$-methacryloyloxyphenyl)-2-pyrazoline] (PMTPP) were reprecipitated from benzene-methanol and THF-methanol, respectively.

Anal. Calcd for $\mathrm{C}_{23} \mathrm{H}_{20} \mathrm{~N}_{2}$ : C, $85.14 \% ; \mathrm{H}, 6.23 \%$; N, 8.64\%. Found for PVTPP: C, 85.02\%; H, $6.23 \%$;
$\mathrm{N}, 8.61 \%$. Anal. Calcd for $\mathrm{C}_{25} \mathrm{H}_{22} \mathrm{~N}_{2} \mathrm{O}_{2}:$ C, $78.51 \%$; $\mathrm{H}, \quad 5.80 \%$; N, $7.32 \%$. Found for PMTPP: C, $78.29 \% ; \quad \mathrm{H}, \quad 5.73 \% ; \quad \mathrm{N}, \quad 7.38 \%$. Copolymerizations were carried out at $60^{\circ} \mathrm{C}$ in a manner similar to the homopolymerization. The total monomer and AIBN concentrations employed were $0.2 \mathrm{M}$ and $2 \times 10^{-3} \mathrm{M}$, respectively, the monomer feed ratio being widely varied. Copolymerizations were stopped at less than $7 \%$ conversion.

\section{Kinetic Studies}

The homopolymerization of the VTPP and MTPP monomers was carried out for various monomer and AIBN concentrations. The polymerization rate was determined from the linear plot of the gravimetrically measured conversion (less than $c a .10 \%$ ) vs. time. The monomer concentration was varied from $5 \times 10^{-2}$ to $4 \times 10^{-1} \mathrm{M}$ for both monomers, and the AIBN concentration was varied from $1 \times 10^{-3}$ to $1.6 \times 10^{-2} \mathrm{M}$ for the VTPP polymerization and from $3 \times 10^{-4}$ to $4 \times 10^{-3} \mathrm{M}$ for the MTPP polymerization. The polymerization temperature was varied from 50 to $80^{\prime \prime} \mathrm{C}$.

\section{Measurements}

IR spectra were taken with a Japan Spectroscopic Co. A-102 grating infrared spectrophotometer. UV absorption spectra were recorded on a Hitachi 124 spectrophotometer. NMR spectra were obtained with a Japan Optics Laboratory $100 \mathrm{MHz}$ JNM-PS100 spectrometer. Mass spectra were taken with a Hitachi RMU-6E spectrometer. Molecular weights were determined with a Waters Associates ALC/GPC 244 high performance liquid chromatographs with THF as the eluent and with a Knawer vapour pressure osmometer with benzene as the solvent.

\section{RESULTS AND DISCUSSION}

\section{Synthesis of New Monomers}

1,3-Diphenyl-5-( $p$-vinylphenyl)-2-pyrazoline (VTPP) was synthesized by the 1,3-dipolar cycloaddition reaction ${ }^{13}$ between $p$-divinylbenzene and $N$ anilinobenzimidoyl chloride in the presence of triethylamine in benzene in a $22-45 \%$ yield. 


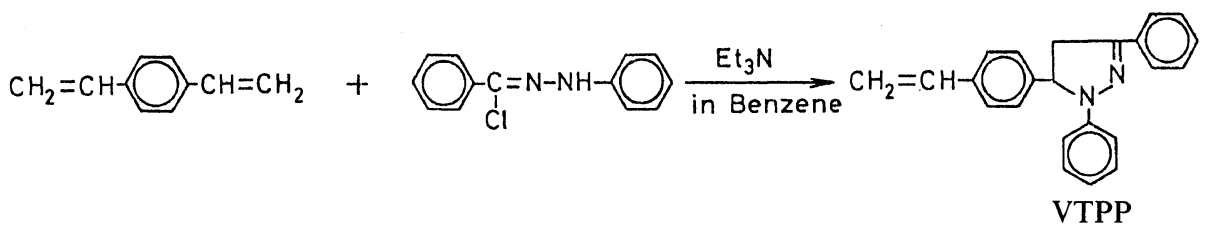

1,3-Diphenyl-5-( $p$-methacryloyloxyphenyl)-2pyrazoline (MTPP) was prepared by the reaction of methacryloyl chloride with 1,3-diphenyl-5-( $p$ - hydroxyphenyl)-2-pyrazoline in the presence of triethylamine in THF in a $55-65 \%$ yield.
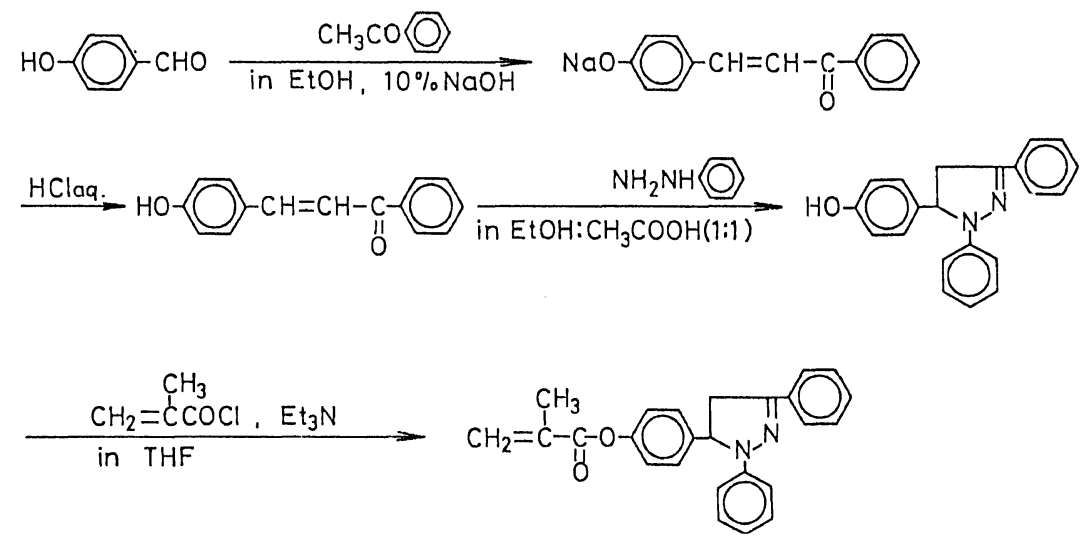

MTPP

These two monomers were identified by the IR, mass and NMR spectra and elementary analyses as described in the experimental section.

\section{Radical Homopolymerization}

The two monomers were polymerized under free radical initiation using AIBN as the initiator to give poly[1,3-diphenyl-5-( $p$-vinylphenyl)-2-pyrazoline] (PVTPP) and poly[1,3-diphenyl-5-( $p$-methacryloyloxyphenyl)-2-pyrazoline] (PMTPP). Typical time-conversion curves for the polymerization of the two monomers are shown in Figure 1.
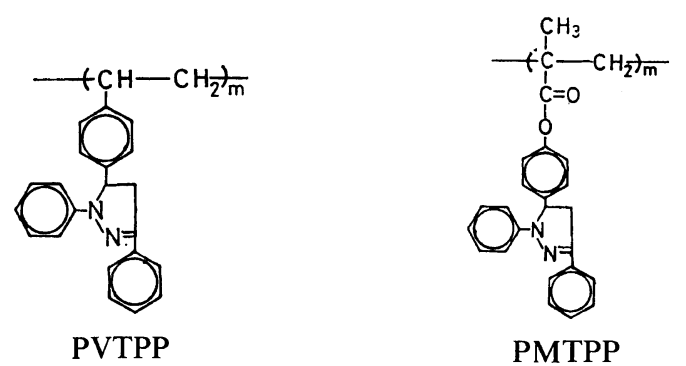

The dependence of the polymerization rate $\left(R_{\mathrm{p}}\right)$ on the monomer and initiator concentrations and on the polymerization temperature was studied for the polymerization of the two monomers. Figures 2, 3 , and 4 show the plots of $\log R_{\mathrm{p}} v s . \log [\mathrm{M}], \log R_{\mathrm{p}}$ vs. $\log [\mathrm{I}]$ and $\ln R_{\mathrm{p}}$ vs. $1 / T$, where [M], [I] and $T$ stands for the monomer and initiator (AIBN) concentrations and the polymerization temperature. The results show that the AIBN-initiated freeradical polymerization of VTPP in benzene at 60 ${ }^{\circ} \mathrm{C}$ is in accord with characteristics of an ideal free radical polymerization, following the relationship $R_{\mathrm{p}}=k_{\mathrm{VTPP}}[\mathrm{M}]^{1.0}[\mathrm{I}]^{0.5}$, and that for the AIBN-initiated free-radical polymerization of MTPP in THF at $60^{\circ} \mathrm{C}$, the kinetic order of monomer is 1.4 , while that of initiator is 0.5 .

Non-ideal kinetic relationships in the free-radical polymerization have been occassionally observed and explained on the basis of several concepts ${ }^{15}$ : (a) complex formation between monomer and initiator, (b) cage effect in the initiation step, (c) primary radical termination, (d) degradative chain transfer and (e) diffusion-controlled termination. The mech- 


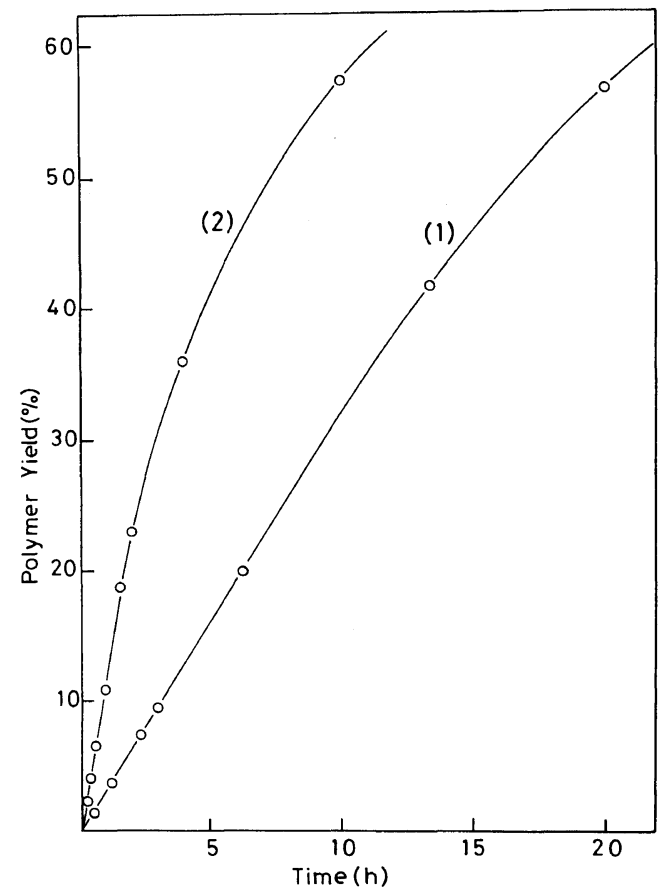

Figure 1. Time-conversion curves for the AIBN-initiated polymerizations of VTPP and MTPP. (1) VTPP in $\mathrm{THF}$ at $60^{\circ} \mathrm{C}$; [VTPP] $=0.2 \mathrm{M},[\mathrm{AIBN}]=2 \times 10^{-3} \mathrm{M}$, (2) MTPP in benzene at $60^{\circ} \mathrm{C} ;[\mathrm{MTPP}]=0.2 \mathrm{M}$, $[\mathrm{AIBN}]=2 \times 10^{-3} \mathrm{M}$.

anism of primary radical termination predicts the kinetic order of initiator being less than 0.5 and that of monomer being greater than $1.0 .^{16}$ The cage effect in the initiation step is considered as a probable explanation for the present relationship $R_{\mathrm{p}}=k_{\mathrm{MTPP}}[\mathrm{M}]^{1.4}[\mathrm{I}]^{0.5}$ for the AIBN-initiated polymerization of MTPP in THF at $60^{\circ} \mathrm{C}^{*}$; however, no further detailed study was made concerning this problem.

* The kinetic relationship of eq 1 holds for the following mechanism, and if $k_{1} \gg k_{2}[\mathrm{M}] \gg k_{3}$, eq 2 is obtained.

$$
\begin{gathered}
\mathrm{I} \underset{k_{1}}{\stackrel{2 k_{\mathrm{d}}}{\rightleftarrows}}(\mathrm{R} \cdot \mathrm{R} \cdot) \stackrel{k_{3}[\mathrm{M}]}{\longrightarrow} \mathrm{M} \cdot+\mathrm{R} \cdot \\
R_{\mathrm{p}}=k_{\mathrm{p}}\left(2 k_{\mathrm{d}} / k_{\mathrm{t}}\right)^{1 / 2}\left(\frac{k_{2}[\mathrm{M}]}{k_{1}+k_{2}[\mathrm{M}]+k_{3}}\right)^{1 / 2}[\mathrm{M}][\mathrm{I}]^{1 / 2} \\
R_{\mathrm{p}}=k_{\mathrm{p}}\left(\frac{2 k_{\mathrm{d}} \cdot k_{2}}{k_{\mathrm{t}} \cdot k_{1}}\right)^{1 / 2}[\mathrm{M}]^{1.5}[\mathrm{I}]^{0.5}
\end{gathered}
$$

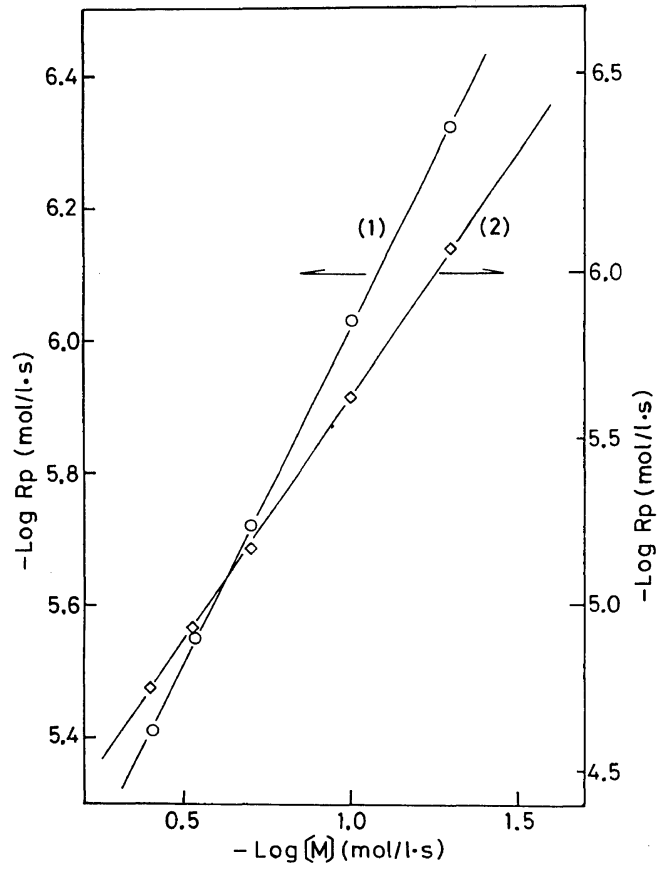

Figure 2. Plot of $\log R_{\mathrm{p}}$ vs. $\log [\mathrm{M}]$ for the AIBNinitiated polymerization of VTPP and MTPP at $60^{\circ} \mathrm{C}$. (1) VTPP in benzene; $[\mathrm{AIBN}]=2 \times 10^{-3} \mathrm{M}$, (2) MTPP in THF; $[$ AIBN $]=2 \times 10^{-3} \mathrm{M}$.

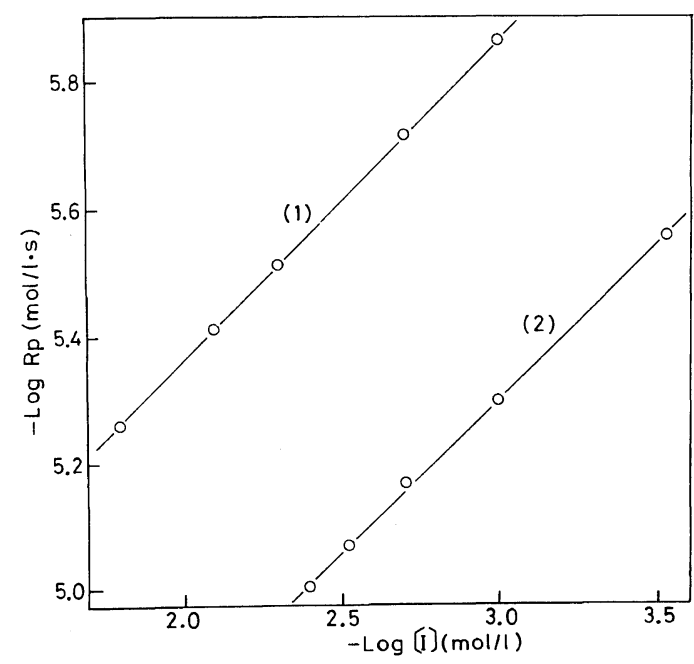

Figure 3. Plot of $\log R_{\mathrm{p}}$ vs. $\log [\mathrm{I}]$ for the AIBNinitiated polymerization of VTPP and MTPP at $60^{\circ} \mathrm{C}$. (1) VTPP in benzene; $[\mathrm{VTPP}]=0.2 \mathrm{M}$, (2) MTPP in THF; [MTPP $]=0.2 \mathrm{M}$. 


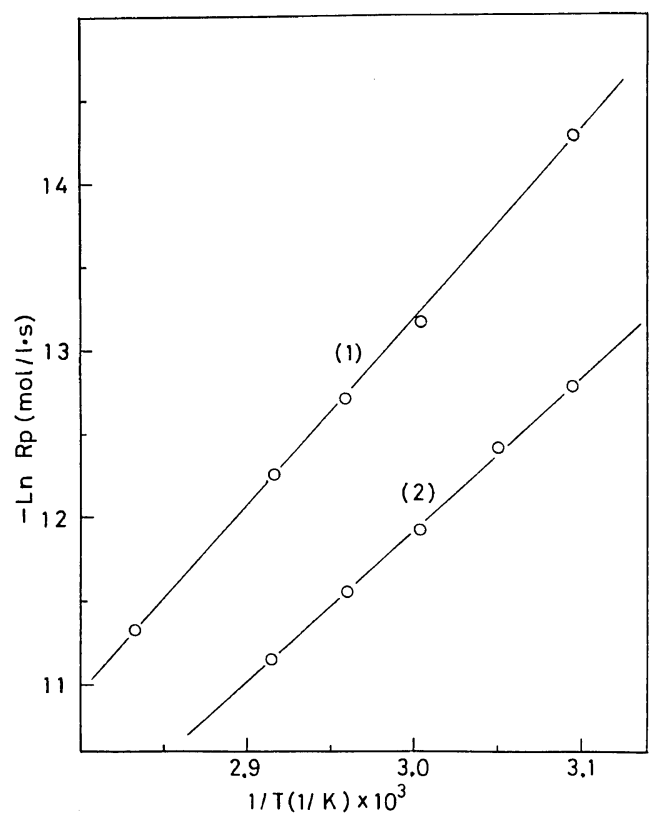

Figure 4. Plot of $\ln R_{\mathrm{p}} v s .1 / T$ for the AIBN-initiated polymerization of VTPP and MTPP. (1) VTPP in benzene; $[\mathrm{VTPP}]=0.2 \mathrm{M},[\mathrm{AIBN}]=2 \times 10^{-3} \mathrm{M}$, (2) MTPP in THF; $[\mathrm{MTPP}]=0.2 \mathrm{M},[\mathrm{AIBN}]=2 \times 10^{-3} \mathrm{M}$.

The value of $k_{\mathrm{VTPP}}$ which equals $k_{\mathrm{p}}\left(2 k_{\mathrm{d}} f / k_{\mathrm{t}}\right)^{1 / 2}$ $\left(k_{\mathrm{p}}, k_{\mathrm{l}}, k_{\mathrm{d}}\right.$, rate constant for the propagation, bimolecular termination and initiator decomposition; $f$, initiation efficiency) for the polymerization of VTPP in benzene at $60^{\circ} \mathrm{C}$ was $2.5 \times 10^{-4} \mathrm{1}^{1 / 2} \mathrm{~mol}^{-1 / 2} \mathrm{~s}^{-1}$ as determined from the intercept value of the linear plot of either Figures 2 or 3 (the values obtained from Figures 2 and 3 were almost the same). Likewise, the value of $k_{\mathrm{MTPP}}$ for the polymerization of MTPP in THF at $60^{\circ} \mathrm{C}$ was obtained as $1.5 \times 10^{-3} \mathrm{l}^{1 / 2} \mathrm{~mol}^{-1 / 2} \mathrm{~s}^{-1}$. The apparent activation energy for the polymerization of VTPP and MTPP was obtained from Figure 4 as 22.3 and $18.3 \mathrm{kcal}$ $\mathrm{mol}^{-1}$, respectively. The literature values of $k_{\mathrm{p}}\left(2 k_{\mathrm{d}} f / k_{\mathrm{t}}\right)^{1 / 2}$ for the AIBN initiated polymerization in benzene at $60^{\circ} \mathrm{C}$ are $6.5 \times 10^{-5}$ for styrene, ${ }^{17} 3.4 \times 10^{-4}$ or $5.3 \times 10^{-4}$ for $\mathrm{MMA}^{18,19}$ and $2.1 \times 10^{-3} 1^{1 / 2} \mathrm{~mol}^{-1 / 2} \mathrm{~s}^{-1}$ for phenyl methacrylate (PhMA). ${ }^{19}$ The apparent overall activation energy for the AIBN-initiated polymerization has been reported to be 21.3 and $22.1 \mathrm{kcal} \mathrm{mol}^{-1}$ for the bulk polymerization of styrene and $p$-methylstyrene ( $p$ MSt) ${ }^{20}$ respectively, and 19.12 and $19.65 \mathrm{kcal}$ $\mathrm{mol}^{-1}$ for the polymerization of $\mathrm{MMA}^{18}$ and

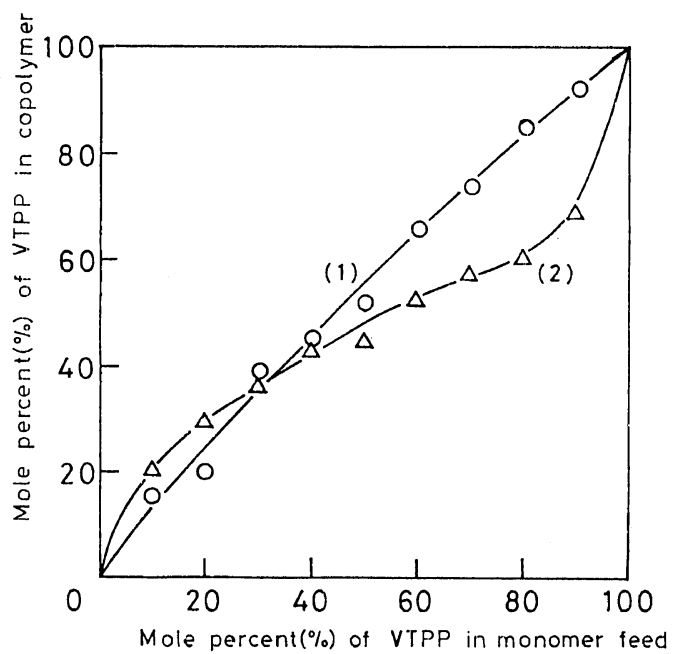

Figure 5. Copolymer composition curves as a function of monomer feed ratios. (1) VTPP-styrene system, (2) MTPP-MMA system; [total monomer] $=0.2 \mathrm{M}$, $[\mathrm{AIBN}]=2 \times 10^{-3} \mathrm{M}$, in benzene at $60^{\circ} \mathrm{C}$.

$\mathrm{PhMA}^{21}$ in benzene, respectively. These results along with the copolymerization results described below indicate that the free-radical polymerizability of the VTPP and MTPP monomers is essentially similar to that of $p$-MSt and PhMA, respectively, although the bulky substituent of TPP may exert some influence on the reactivity of VTPP and MTPP.

\section{Radical Copolymerization}

The properties of functional polymers can be varied by the method of copolymerization with appropriate comonomers. In addition, copolymers with varying composition serve as materials for studying polymer effects on properties and functions. The VTPP and MTPP monomers were copolymerized with styrene, MMA, fumaronitrile, and diethyl fumarate to give corresponding copolymers, and $Q$ and $e$ values as well as the monomer reactivity ratios were determined for the present new monomers. Figures 5 and 6 show the copolymer composition curves as a function of monomer feed ratios for the AIBN-initiated copolymerizations of VTPP with styrene and MMA, and those of MTPP with styrene, MMA, fumaronitrile, and diethyl fumarate, respectively, as determined from the nitrogen content.

Monomer reactivity ratios were determined from the Fineman-Ross plot using the least-squares- 


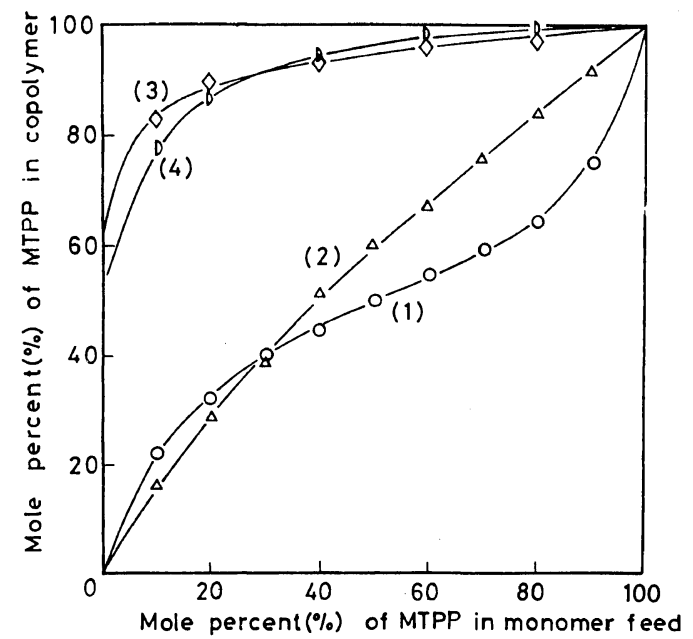

Figure 6. Copolymer Composition curves as a function of monomer feed ratios. (1) MTPP-styrene system, (2) MTPP-MMA system, (3) MTPP-fumaronitrile system, (4) MTPP-diethyl fumarate system; [total monomer $]=0.2 \mathrm{M},[\mathrm{AIBN}]=2 \times 10^{-3} \mathrm{M}$, in $\mathrm{THF}$ at 60 .

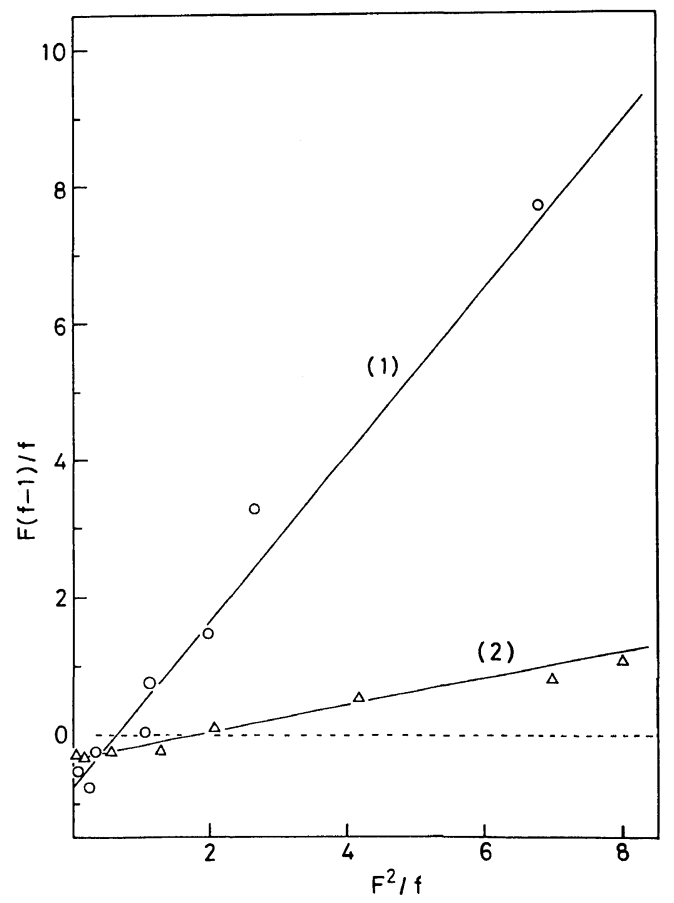

Figure 7. Fineman-Ross plot for the copolymerizations of VTPP with styrene (1) and MMA (2).

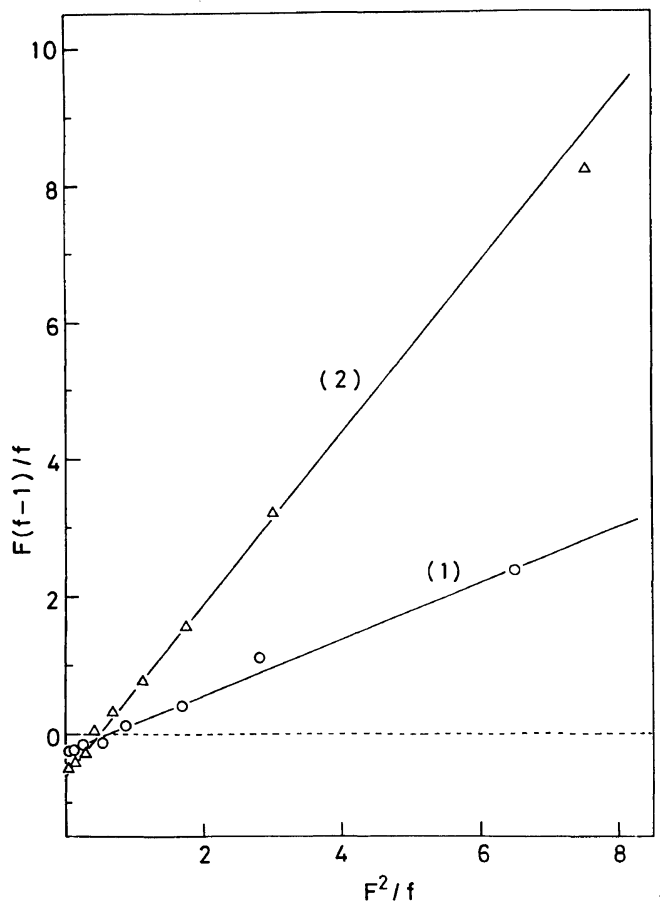

Figure 8. Fineman-Ross plot for the copolymerizations of MTPP with styrene (1) and MMA (2).

method (Figures 7 and 8). Tables I and II list the monomer reactivity ratios and the $Q$ and $e$ values of VTPP and MTPP, respectively, with reference to the literature data for $p$-MSt and PhMA. The results show that the radical copolymerizability of VTPP and MTPP resembles $p$-MSt and PhMA, respectively.

\section{Characterization of Polymers}

The structure of the polymers was identified as the corresponding vinyl and methacrylate polymer based on the IR, ${ }^{1} \mathrm{H}$ NMR and electronic absorption spectra and elementary analyses. The characteristic IR absorption bands observed at 1622 $\mathrm{cm}^{-1}$ for VTPP and $1630 \mathrm{~cm}^{-1}$ for MTPP due to the $\mathrm{C}=\mathrm{C}$ stretching vibration and those at 910 and $990 \mathrm{~cm}^{-1}$ for VTPP and $943 \mathrm{~cm}^{-1}$ for MTPP due to the vinylic $\mathrm{C}-\mathrm{H}$ out-of-plane deformation vibration disappeared in their homo- and copolymers. The electronic absorption spectra of the homo- and copolymers of VTPP and MTPP show the intense band with $\lambda_{\max }$ at 362 and $359 \mathrm{~nm}$ in benzene, respectively, due to the intramolecular charge- 
Table I. Monomer reactivity ratios $\left(r_{1}, r_{2}\right)$ and $Q$ and $e$ values for the VTPP monomer

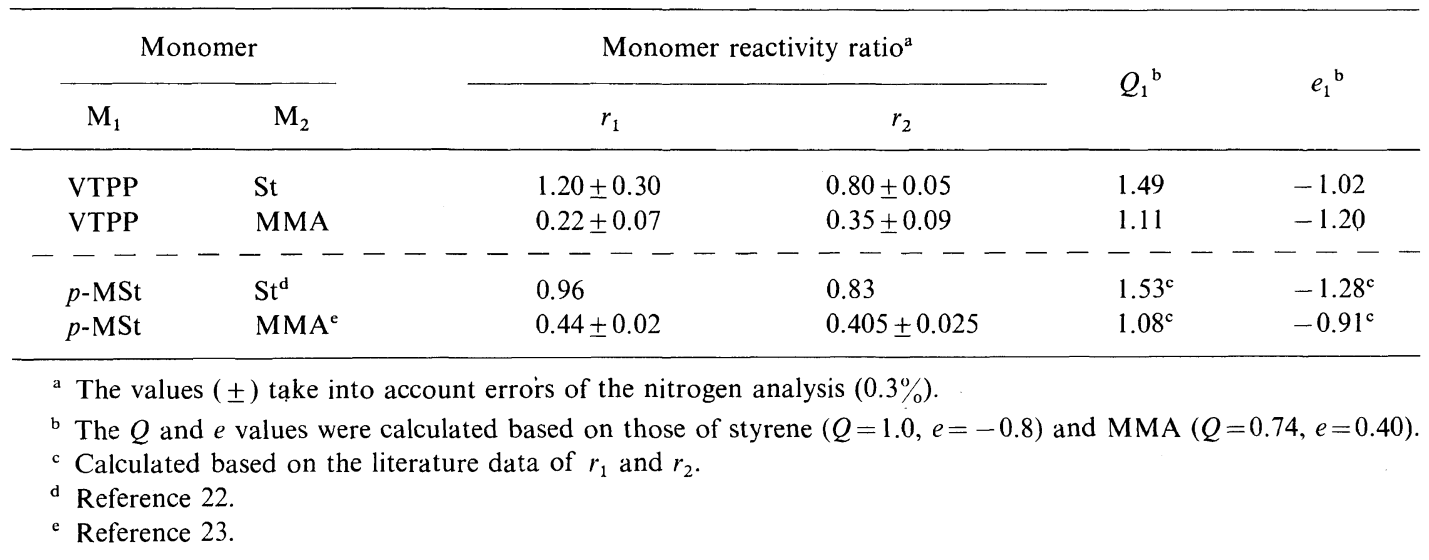

Table II. Monomer reactivity ratios $\left(r_{1}, r_{2}\right)$ and $Q$ and $e$ values for the MTPP monomer

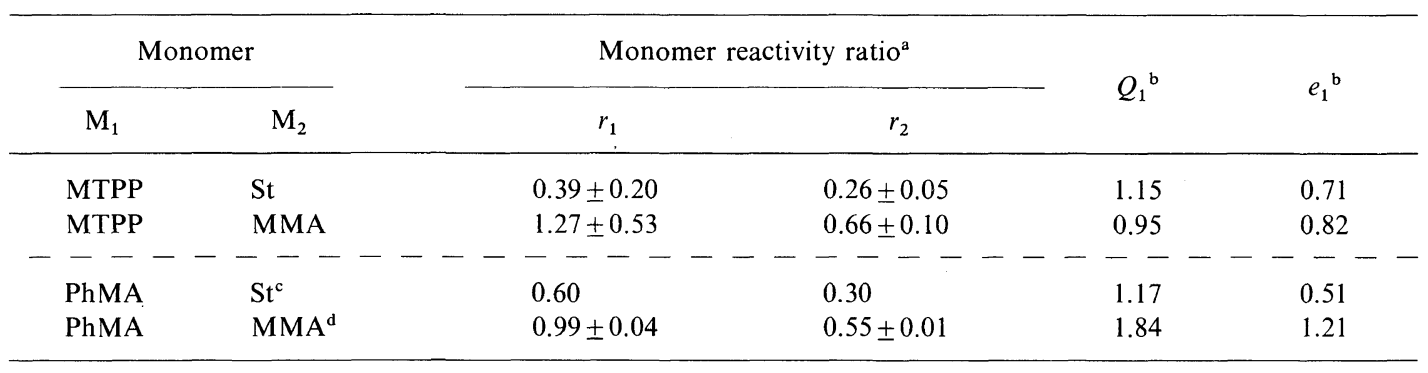

a The values $( \pm)$ take into account errors of the nitrogen analysis $(0.3 \%)$.

b The $Q$ and $e$ values were calculated based on those of styrene $(Q=1.0, e=-0.8)$ and MMA $(Q=0.74, e=0.40)$.

c Reference 24.

d Reference 25.

transfer band of the TPP group. These results indicate that the polymerization proceeded at the $\mathrm{C}=\mathrm{C}$ double bond without any destruction of the TPP moiety. A noticeable observation in the ${ }^{1} \mathrm{H}$ NMR spectra of PVTPP and PMTPP is that the chemical shift of one of the phenyl protons of the TPP ring is shifted upfield to $\delta 6.4 \mathrm{ppm}$ in PVTPP compared with the spectra of TPP and PMTPP as shown in Figure 9, suggesting that some mutual interactions exist between neighbouring TPP groups in PVTPP in contrast to PMTPP where pendant TPP groups are further away from the main chain.

The homo- and copolymers of VTPP and MTPP were easily soluble in THF and $N, N$-dimethylformamide, soluble in benzene, 1,2-dichloroethane and dioxane, partly soluble in acetone, ethyl ace- tate, but insoluble in hexane, diethyl ether, and alcohol. The number-average molecular weight $\left(M_{n}\right)$ of PVTPP and PMTPP was in the range from 9,000 to 18,000 and from 10,000 to 25,000 , respectively, depending upon the polymerization conditions. As expected, $M_{n}$ increased with an increase in the monomer concentration and a decrease in the initiator concentration and polymerization temperature. The values of $M_{n}$ for the copolymers of VTPP and MTPP were smaller than those of the corresponding homopolymers, ranging from $c a$. 3,000 to 10,000. Examples are given of the values of $M_{n}$ and $M_{w}$ for PVTPP and PMTPP : PVTPP (obtained under conditions $[\mathrm{M}]=0.35 \mathrm{M}$, $[\mathrm{AIBN}]=9 \times 10^{-4} \mathrm{M}$, in benzene at $55^{\prime \prime} \mathrm{C}$ ): $M_{n}=18,000, \quad M_{w}=31,000, \quad M_{w} / M_{n}=1.72 \quad(\mathrm{GPC}$ 


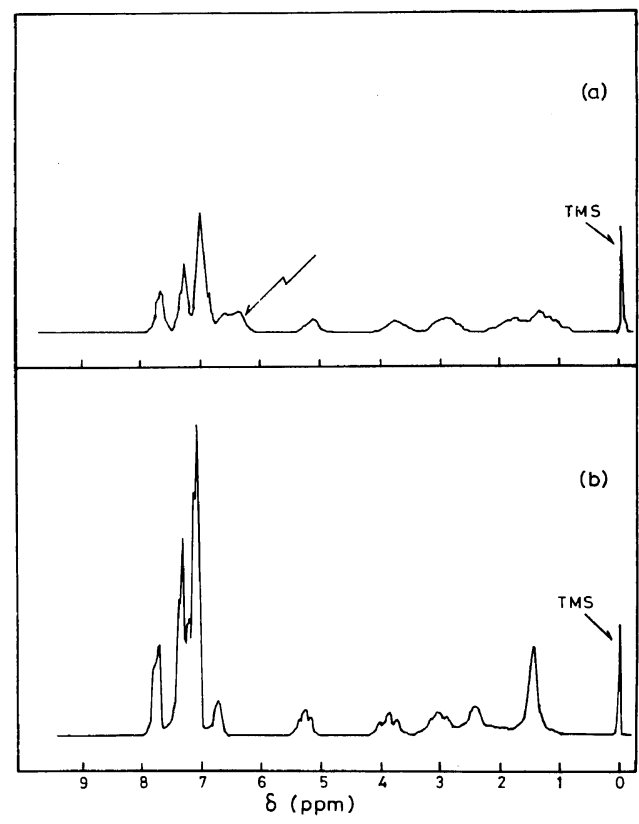

Figure 9. ' $1 \mathrm{H}$ NMR spectra of PVTPP (a) an PMTPP (b) in $N, N^{\prime}$-dimethylformamide- $d_{7}$ at $120^{\circ} \mathrm{C}$.

with polystyrene as a standard), $M_{n}=16,000$ (VPO); PMTPP (obtained under conditions $[\mathrm{M}]=0.6 \mathrm{M}$, $[\mathrm{AIBN}]=1 \times 10^{-3} \mathrm{M}$, in THF at $\left.50^{\circ} \mathrm{C}\right): M_{n}=$ 25,000, $M_{w}=43,000, \quad M_{w} / M_{n}=1.72 \quad(\mathrm{GPC}$ with polystyrene as a standard), $M_{n}=23,000$ (VPO). The homopolymers, PVTPP and PMTPP, were thermally stable below $200^{\circ} \mathrm{C}$, softening at ca. 205 and $209^{\circ} \mathrm{C}$ respectively. Thermogravimetric analyses of PVTPP and PMTPP showed that these homopolymers started to lose weight at $c a .350^{\circ} \mathrm{C}$, PVTPP being a little bit thermally more stable than PMTPP as shown in Figure 10.

Acknowledgement. The authors wish to thank the Research Laboratory of Nippon Sheet Glass Co., Ltd. for making the GPC measurements.

\section{REFERENCES}

1. P. J. Melz, R. B. Champ, L. S. Chang, C. Chiou, G. S. Keller, L. C. Liclican, R. R. Neiman, M. D. Shattuck, and W. J. Weiche, Photo. Sci. Eng., 21, 73 (1977).

2. I. H. Leaver and V. E. Rivett, Mol. Photochem., 6, 113 (1974).

3. H. Strahle, W. Sertz, and H. Guster, Ber Bunsenges.

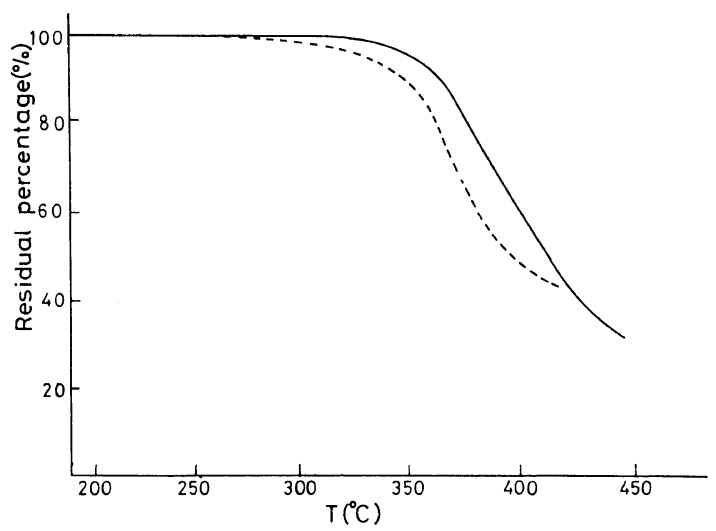

Figure 10. TG curves for PVTPP (-), and PMTPP $(----)$.

Phys. Chem., 80, 288 (1976).

4. A. Wagner, C. W. Schellhammer, and S. Peterson, Angew. Chem. Int. Ed., 5, 699 (1966).

5. H. Yamamoto, Y. Sano, Y. Shirota, H. Seki, and H. Mikawa, Bull. Chem. Soc. Jpn., 52, 1533 (1978).

6. Y. Sano, M. Yokoyama, Y. Shirota, and H. Mikawa, Mol. Cryst. Liq. Cryst., 53, 291 (1979).

7. Y. Sano, Y. Shirota, and H. Mikawa, Bull. Chem. Soc. Jpn., 52, 2192 (1979).

8. K. Morimoto, Y. Hayashi, and A. Inami, Bull. Chem. Soc. Jpn., 36, 1651 (1977).

9. H. Tsukahara, T. Krita, and K. Futaki, Nippon Shashin Gakkai Kaishi (Japan), 29, 12 (1966).

10. F. B. Kaufman and E. M. Engler, J. Am. Chem. Soc., 101, 547 (1979); ibid., 102, 483 (1980).

11. F. Iinuma, H. Mikawa, and Y. Shirota, Int. J. Polym. Photochem.; Macromolecules; Mol. Cryst. Liq. Cryst., in press.

12. B. T. Storey, J. Polym. Sci., A3, 265 (1965).

13. R. Huisgen, M. Seidel, G. Wallbillich, and H. Kunpfer, Tetrahedron, 17, 3 (1962).

14. D. Volander, Berichte, 58, 127 (1925).

15. G. E. Scott and E. Senogles, J. Macromol. Sci. Rev. Macromol. Chem., C(9), 49 (1973).

16. P. C. Deband and I. D. Gaba, Makromol. Chem., 179, 1549 (1978).

17. J. C. Bevington, Trans. Faraday Soc., 51, 1392 (1955).

18. P. C. Debb and S. K. Kapoor, Eur. Polym. J., 15, 477 (1979).

19. G. Ayrey and A. C. Yaynes, Eur. Polym. J., 9, 1029 (1973).

20. M. Imoto, M. Kinoshita, and M. Nishigaki, Makromol. Chem., 86, 217 (1965).

21. H. Boudevska and V. Popminchev, Makromol. Chem., 143, 19 (1971).

22. A. V. Chemobai and Zh. Kh. Zelichenko, 


\section{F. IINUma, H. Mikawa, and Y. Shirota}

Vysokomol. Soedin., Ser. A, 11, 1470 (1969).

23. C. Walling, F. R. Briggs, and K. B. Wolfstirn, J. Am. 25. M. Kamachi, D. J. Liaw, and S. Nozakura, Polym. Chem. Soc., 70, 1537 (1948).

24. T. Otsu, T. Ito, and M. Imoto, J. Polym. Sci., B, 3,
113 (1965).

J., 10, 641 (1978). 